HORACE

THE ODES 



\section{FACING PAGES}

NICHOLAS JENKINS

Series Editor 


\section{EDITED BY J. D. MCCLATCHY}

$\begin{array}{rr}\text { New translations by } & \text { James Lasdun } \\ \text { Eavan Boland } & \text { J. D. McClatchy } \\ \text { Robert Creeley } & \text { Heather McHugh } \\ \text { Dick Davis } & \text { W. S. Merwin } \\ \text { Mark Doty } & \text { Paul Muldoon } \\ \text { Alice Fulton } & \text { Carl Phillips } \\ \text { Debora Greger } & \text { Robert Pinsky } \\ \text { Linda Gregerson } & \text { Marie Ponsot } \\ \text { Rachel Hadas } & \text { Charles Simic } \\ \text { Donald Hall } & \text { Mark Strand } \\ \text { Robert Hass } & \text { Charles Tomlinson } \\ \text { Anthony Hecht } & \text { Ellen Bryant Voigt } \\ \text { Daryl Hine } & \text { David Wagoner } \\ \text { John Hollander } & \text { Rosanna Warren } \\ \text { Richard Howard } & \text { Richard Wilbur } \\ \text { John Kinsella } & \text { C. K. Williams } \\ \text { Carolyn Kizer } & \text { Charles Wright } \\ & \text { Stephen Yenser }\end{array}$




\section{HORACE \\ THE ODES}

New Translations by Contemporary Poets

PRINCETON UNIVERSITY PRESS

Princeton and Oxford 
Copyright $\odot 2002$ by Princeton University Press

Published by Princeton University Press, 41 William Street, Princeton,

New Jersey 08540

In the United Kingdom: Princeton University Press, 3 Market Place,

Woodstock, Oxfordshire OX20 1SY

All Rights Reserved

Third printing, and first paperback printing, 2005

Paperback ISBN 0-691-11981-3

The Library of Congress has cataloged the cloth edition of this book as follows

Horace.

Horace, the Odes / new translations by contemporary poets, Robert Bly ... [et al.] ; edited by J. D. McClatchy.

p. cm. - (Facing pages)

Includes index.

ISBN 0-691-04919-X (cloth : alk. paper)

1. Horace-Translations into English. 2. Laudatory poetry, Latin-Translations into English. I. Bly, Robert. II. McClatchy, J. D., 1945- III. Title. IV. Series. PA6394 .A2 2002

$874^{\prime} .01-\mathrm{dc} 21 \quad 2002023128$

British Library Cataloging-in-Publication Data is available

This book is supported by the Charles Lacy Lockert Fund of Princeton University Press.

This book has been composed in Akzidenz-Grotesk and Minion

Odes I.16, I.26, II.3, and II.9 ๑ Robert Bly.

Odes I.9, I.35, II.14, III.1, III.30, IV.9 @ John Hollander.

Ode III.23 “An Offering," from Waterborne by Linda Gregerson. Copyright $\odot 2002$ by Linda

Gregerson. Reprinted by permission of Houghton Mifflin Company. All rights reserved.

"Horace: Ode I.34," "Horace: Ode I.37," from Shadow of Heaven by Ellen Bryant

Voigt. Copyright $\odot 2002$ by Ellen Bryant Voigt. Used by permission of W. W. Norton \&

Company, Inc.

Printed on acid-free paper. $\infty$

pup.princeton.edu

Printed in the United States of America

109876543 